\title{
Etude comparative des performances zootechniques d'une souche domestiquée de Clarias gariepinus (Burchell, 1822) et d'une souche sauvage de Clarias anguillaris (Linnaeus, 1758)
}

\author{
Inoussa COMPAORE $^{1 *}$, Aboubacar TOGUYENI ${ }^{1,2}$, Carole ROUGEOT $^{3}$, \\ Patrick KESTEMONT ${ }^{4}$ et Charles MELARD ${ }^{3}$
}

\footnotetext{
${ }^{1}$ Unité de Recherche Aquaculture et Biodiversité Aquatique/Laboratoire d'Etude et de Recherche sur les Ressources Naturelles et Sciences de l'Environnement (UR-ABAQ/LERNSE), Institut du Développement Rural (IDR), Université Polytechnique de Bobo-Dioulasso (UPB) ; 01 BP 1091 Bobo-Dioulasso 01, Burkina Faso. ${ }^{2}$ Centre International de Recherche-Développement sur l'élevage en zone Subhumide (CIRDES), 01 BP 454 Bobo-Dioulasso 01, Burkina Faso.

${ }^{3}$ Centre de Formation et de Recherche en Aquaculture (CEFRA), Université de Liège; 10, chemin de la justice, B-4500 Tihange, Belgique.

${ }^{4}$ Research Unit in Environmental and Evolutionary Biology, Université de Namur ; Rue de Bruxelles 61-5000; Namur, Belgique.

*Auteur correspondant ; E-mail: inoussacompaore@yahoo.fr
}

\section{RESUME}

Les deux espèces (Clarias gariepinus et C. anguillaris) du sous-genre Clarias (clarias) ont été identifiées parmi l'ichtyofaune du Burkina Faso. L'analyse de leur structuration fait état de la prédominance de C. anguillaris. Mais, pour l'instant, cette dernière espèce n'a pas encore fait l'objet de domestication. L'objectif de cette étude est d'évaluer les performances zootechniques de cette espèce en comparaison avec $C$. gariepinus domestiquée. L'étude est réalisée sur 3 familles de chacune des deux espèces. Chaque famille est obtenue par croisement d'un male et d'une femelle. Les alevins, obtenus via une reproduction artificielle, sont repartis dans six (6) aquariums de 50 litres maintenus en circuit fermé, à un poids moyen individuel de $1 \mathrm{~g}$. Deux densités de mise en charge de 2 et 4 poissons/litres ont été utilisées en réplicas. Les poissons sont élevés pendant 30 jours. Les résultats montrent que chez C. gariepinus le poids moyen individuel a varié entre 35,37 et $42,18 \mathrm{~g}$ et la longueur totale entre 173,5 et $187,55 \mathrm{~mm}$; le taux de conversion alimentaire de 0,70 et 0,82 ; le taux de survie de 47,87 à $66,62 \%$. Chez $C$. anguillaris le poids moyen individuel est compris entre 11,68 et $29,13 \mathrm{~g}$ et la longueur totale entre 128,4 et $159,2 \mathrm{~mm}$; le taux de conversion alimentaire de 0,71 et 1,28 tandis que le taux de survie a fluctué entre 53 et $68,87 \%$. Ces résultats préliminaires montrent que $C$. anguillaris peut avoir des performances de croissance proches de celles de $C$. gariepinus et pourrait donc être utilisée en aquaculture.

(C) 2016 International Formulae Group. All rights reserved.

Mot clés : C. gariepinus, C. anguillaris, domestication, performance de croissance, Burkina Faso. 


\title{
Comparative study of growth performance of a domesticated strain of Clarias gariepinus (Burchell, 1822) and a wild strain of Clarias anguillaris (Linnaeus, 1758)
}

\begin{abstract}
Both species (Clarias gariepinus and C. anguillaris) subgenus Clarias (catfish) were identified among the fish fauna of Burkina Faso. Analysis of their structuring reported the prevalence of $C$. anguillaris. But for now, the latter species has not yet been the subject of domestication. The aim of this study is to assess the zootechnical performance of this species compared with domesticated strain of C. gariepinus. The study was conducted on 3 families of each species. Each family is obtained by crossing a male and a female. The fry, obtained through artificial reproduction, are divided into six (6) 50 liter aquariums, kept in closed circuit at $1 \mathrm{~g}$ individual mean weight. Two densities ( 2 to 4 fish / liter) were used in replicas. The fish are raised for 30 days. The results showed that in C. gariepinus the mean individual weight ranged between 35.37 and $42.18 \mathrm{~g}$ and the total length between 173.5 and $187.55 \mathrm{~mm}$; feed conversion rate of 0.70 and 0.82 ; the survival rate of 47.87 to $66.62 \%$. In C. anguillaris individual mean weight is between 11.68 and $29.13 \mathrm{~g}$ and the total length between 128.4 and $159.2 \mathrm{~mm}$; the feed conversion ratio of 0.71 and 1.28 while the survival rate has fluctuated between 53 and $68.87 \%$. These preliminary results show that $C$. anguillaris could have growth performance similar to those of $C$. gariepinus and could therefore be used in aquaculture.
\end{abstract}

(c) 2016 International Formulae Group. All rights reserved.

Keywords: C. gariepinus, C. anguillaris, domestication, growth performance, Burkina Faso.

\section{INTRODUCTION}

Les poisson-chats africains contribuent pour une part très importante ces dernières années à la production totale de poissons d'élevage en Afrique sub-saharienne et dans certains pays asiatiques (Hecht, 2013). L'espèce Clarias gariepinus, reconnue comme ayant un taux de croissance élevé, une grande résistance aux manipulations et aux stress, et très bien appréciée dans un grand nombre de pays, est la plus utilisée. C'est dans les années 1970 à 1980 que l'on commence à maîtriser les techniques de base pour la reproduction artificielle de l'espèce Clarias gariepinus (Burchell, 1822), l'alevinage et le grossissement par alimentation artificielle (Hecht, 2013). Ces travaux ont été poursuivis jusqu'à une date récente en vue de répondre aux exigences des nouvelles conditions d'élevage (Hossain et al., 2001 ; Eding and Kamstra, 2002 ; Ducarme et Micha, 2003 ; Rukera Tabaro et al., 2005; Eding et al., 2006). C'est cette donnée majeure qui a permis le développement de la pisciculture semi-intensive puis intensive de cette espèce.
En 2010, la production de cette espèce a atteint $453 \quad 209$ et $198 \quad 286$ tonnes respectivement pour l'Asie et l'Afrique subsaharienne (FAO, 2012).

Au Burkina Faso, l'élevage du poisson chat est encore à ces débuts, la production piscicole repose encore, pour une bonne part, sur l'élevage du tilapia du Nil (Oreochromis niloticus). Il est toutefois utilisé comme prédateur dans les étangs de tilapia et la collecte des juvéniles se fait dans le milieu naturel, ce qui compromet sa survie à long terme. Avant les travaux sur la caractérisation morphologique (Compaoré et al., 2015), seule l'espèce C. gariepinus était mentionnée dans les statistiques. Les résultats de ces études ont révélé l'existence des deux espèces $(C$. gariepinus et $C$. anguillaris) du sous-genre Clarias (Clarias) avec une forte prédominance de $C$. anguillaris sur l'ensemble du réseau hydrographique du pays.

Les données de la littérature (Paugy et al., 2003 ; Compaoré et al., 2015) révèlent qu'en milieu naturel, des individus de plus de $500 \mathrm{~mm}$ de longueur totale sont retrouvés 
chez les deux espèces. Ce qui laisse penser qu'en milieu contrôlé (élevage), ces deux espèces pourraient avoir une croissance similaire. Cependant, il n'existe pratiquement pas de données sur des études comparatives des performances de croissance de ces espèces en milieu contrôlé. Dans le cadre de la promotion du développement de l'aquaculture, la diversification des espèces constitue un moyen privilégié, surtout si elle se repose sur l'utilisation d'espèces autochtones ayant de fort potentiel de croissance. La présente étude a pour but d'évaluer et de comparer les performances de croissance des deux espèces dont l'une $(C$. gariepinus) est domestiquée et utilisée dans des fermes de production à grande échelle et l'autre $(C$. anguillaris) issue du milieu naturel et donc n'ayant jamais fait l'objet de domestication. Mais vu sa prédominance dans les cours d'eau du pays, la domestication et la caractérisation des performances zootechniques se révèlent être une priorité pour le développement de la pisciculture. Il s'agit de façon spécifique de tester les performances zootechniques de $C$. anguillaris en comparaison avec une souche domestiquée de $C$. gariepinus en condition entièrement contrôlé.

\section{MATERIEL ET METHODES}

\section{Structure d'élevage}

Les expériences ont été conduites dans un circuit fermé de 32 aquariums de 50 litres chacun, avec une photopériode de $12 \mathrm{D} / 12 \mathrm{~N}$ (diurne/nocturne) au sein de l'Unité de Recherche Aquaculture et Biodiversité Aquatique (UR-ABAQ) de l'Université Polytechnique de Bobo-Dioulasso.

\section{Matériel biologique}

Le matériel biologique est constitué de 3 familles de souche domestiquée de $C$. gariepinus et de 3 familles de souche sauvage de $C$. anguillaris. La souche domestiquée provient de la ferme d'aquaculture de TONON au Bénin. Quant à la souche sauvage de $C$. anguillaris, elle a été capturée en milieu naturel dans le barrage de Bagré au Burkina Faso. Les reproductions sont réalisées par induction de la ponte avec de l'Ovaprim (hormone de synthèse composée de GnRH analogue de saumon et d'un inhibiteur de la dopamine) suivi d'une fécondation et d'une incubation artificielle des œufs.

\section{Elevage larvaire et mise en charge des alevins \\ Les larves de chaque espèce sont} élevées jusqu'à $1 \mathrm{~g}$ environ de poids moyen individuel (pmi) avant d'être mis en charge pour le suivi de la croissance. Deux densités de mise en charge ( 2 et 4 poissons/litres, soit 2000 et 4000 alevins $/ \mathrm{m}^{3}$ ) sont utilisées pour chacune des espèces. Ces mises en charges se réfèrent aux densités de mise en charge recommandées en phase de pré-grossissement (De Graaf et Janssen, 1996). Chaque densité est répétée 2 fois (Tableau 1). Au cours de l'expérience, les contrôles (taux de survie, longueur et poids) sont effectués tous les 10 jours.

\section{Contrôle des paramètres physico-chimiques}

Les paramètres physico-chimiques (température, $\mathrm{pH}$, conductivité et oxygène dissous) sont pris dans les aquariums deux fois par jour, le matin à 8 heures et l'aprèsmidi à 15 heures. La première mesure est effectuée avant le premier nourrissage et la seconde avant le dernier nourrissage.

\section{Nourrissage}

Les larves sont nourries d'abord avec des proies vivantes (artémia) puis avec deux d'aliments commerciaux (M-150 Gemma Micro et Me-0,5 Crumble Start) successivement et ce jusqu'à avoir un poids moyen individuel (pmi) de $1 \mathrm{~g}$ avant d'être mises en charge pour l'expérimentation. Pendant les 30 jours d'élevage, les alevins ont été nourris dans un premier temps avec de l'aliment commercial Skretting de $1 \mathrm{~mm}$ (Me$1,0 \mathrm{~mm}$ MP Starter) de diamètre et dans un second temps avec 1,8 mm (Me-1,8 mm P Starter). Ceci a but d'adapter la taille de 
l'aliment en fonction de la croissance du poisson. La composition des aliments est donnée dans le Tableau 2.

\section{Paramètres zootechniques}

Le contrôle de la croissance est réalisé de façon périodique tous les 10 jours. A chaque contrôle, les paramètres suivants sont notés : la biomasse totale, le nombre d'individus, la longueur totale, le poids individuel et la quantité d'aliment distribuée afin de calculer les principaux indices d'évaluation de la performance de chaque espèce (Tableau 3 ).

\section{Analyse des données}

Le test de Newman-Keuls a été utilisé pour identifier les groupes homogènes des différents paramètres tels que : le poids moyen individuel, le gain moyen quotidien, le taux de conversion alimentaire (TCA), la longueur totale (LT), le taux de survie (TS), les taux de croissance spécifique (TCS) et le coefficient de condition $(\mathrm{K})$. Ces tests ont été réalisés à l'aide du logiciel Statistica version 7.1. L'hypothèse nulle est rejetée au seuil de $5 \%$. Les graphiques ont été générés grâce au logiciel Microsoft Excel 2016.

\section{RÉSULTATS}

\section{Paramètres physico-chimiques}

L'évolution des paramètres physicochimiques est présentée dans le Tableau 4. L'analyse du tableau montre qu'en dehors du $\mathrm{PH}$, les autres paramètres présentent des faibles valeurs dans la matinée que celles du soir. Un test Univarié (MANOVA) montre qu'il existe une différence hautement significative au seuil de 5\% entre les valeurs matinales et celles du soir.

\section{Evolution du taux de survie}

L'évolution des taux de survie au cours de l'expérimentation pour chacune des 3 familles de chaque espèce est présentée dans la Figure 1. Ces taux ont varié de $47,87 \%$ à $68,87 \%$ après 30 jours d'élevage. Les familles présentant les meilleurs taux de survie $(>60 \%)$ sont les F1 et F3 de C. anguillaris et les F2 et F3 de C. gariepinus. Quant aux familles F2 et $\mathrm{F} 1$ de $C$. anguillaris et $C$. gariepinus les taux de survie sont plus faibles $(<60 \%)$. Cependant, l'analyse de variance (ANOVA) n'a pas révélé de différence significative ( $p>0,05)$ entre toutes ces familles.

\section{Evolution du poids moyen individuel}

L'évolution des poids moyens individuels (pmi) des 3 familles de chaque espèce est représentée dans la Figure 2. L'analyse du graphique fait ressortir que les 3 familles (F1, F2 et F3) de C. gariepinus présentent les meilleures croissances. Leurs poids moyens varient de 35,37 à 42,18. Pour les familles de $C$. anguillaris, c'est la F3 qui présente la meilleure croissance $(29,13)$ avec des valeurs proches de celles des familles de C. gariepinus. Les $\mathrm{F} 1$ et $\mathrm{F} 2$ ont eu des croissances significativement plus faible (ANOVA, $\mathrm{p}<0,05)$ et cela dès les 10 premiers jours d'élevage. L'ANOVA fait ressortir également une différence significative $(\mathrm{p}<0,05)$ de croissance entre la F2 de $C$. gariepinus et la F1 de $C$. anguillaris dès la pêche de contrôle à 20 jours d'élevage.

\section{Evolution de la longueur totale}

La Figure 3 présente l'évolution de la taille des poissons exprimée par la longueur totale $(\mathrm{mm})$ pour chaque famille. A la fin de l'expérimentation (30 jours d'élevage) la taille moyenne observée chez $C$. gariepinus est comprise entre 173,5 et $187,55 \mathrm{~mm}$ tandis qu'elle fluctue entre 128,4 et $159,2 \mathrm{~mm}$ chez C. anguillaris. Ces résultats montrent ainsi une bonne croissance en longueur de $C$. gariepinus comparativement à $C$. anguillaris. Le test de Newman-Keuls met en évidence des différences significatives $(p<0,05)$ entre les différentes familles des deux espèces. Ainsi, on peut distinguer 4 groupes $(\mathrm{G} 1=\mathrm{F} 1$ de $C$. anguillaris, $\mathrm{G} 2=\mathrm{F} 2$ et $\mathrm{F} 3$ de $C$. anguillaris, G3= F1 et F3 de $C$. gariepinus et $\mathrm{G} 4=\mathrm{F} 2$ de $C$. gariepinus). 


\section{Comparaison des performances} zootechniques des deux espèces

A la fin de l'expérimentation (30 jours d'élevage), le gain moyen quotidien (GMQ) observé chez l'ensemble des deux espèces varie de 0,47 à 1,38 (Tableau 5). Les 3 familles de $C$. gariepinus enregistrent les meilleurs gains avec des valeurs supérieures à 1. Ensuite vient les gains les plus faibles sont observés au niveau des familles F1 et F2 de $C$. anguillaris qui diffèrent statistiquement (p<0,05) des autres. La famille F3 de $C$. anguillaris est intermédiaire entre les deux groupes.

Le taux de conversion alimentaire (TCA) varie de 0,7 à 1,28 et les meilleurs taux (<1) sont observés au niveau des familles F1, F2, F3 de $C$. gariepinus et F3 de $C$. anguillaris. Ces familles diffèrent statistiquement (test de Newman-Keuls, $\mathrm{p}<0,05)$ de $\mathrm{F} 1$ et $\mathrm{F} 2$ de $C$. anguillaris.

Le taux de croissance spécifique pondérale (TCSP) est très hétérogène d'une famille à une autre. Il varie de 8,08 à 12,02 et les valeurs les plus élevées sont observées au niveau de F1 et de F2 de C. gariepinus. Les valeurs les plus faibles sont enregistrées chez
$\mathrm{F} 1$, F2 de $C$. anguillaris qui différent statistiquement $(\mathrm{p}<0,05)$ des autres familles. Quant au coefficient de condition $\mathrm{K}$, des valeurs sont comprises entre 0,44 et 0,97 . La quasi-totalité des familles sont statistiquement identiques à l'exception de la famille F2 de $C$. anguillaris.

\section{Effet de la densité sur le taux de survie et la croissance}

Les données de comparaison de l'effet de la densité ( 2 ou 4 poissons/litre) sur le taux de survie durant la période d'expérimentation sont présentées dans le Tableau 6. Malgré une hétérogénéité observée entre les aquariums avec les deux densités, le test de NewmanKeuls ne montre aucune différence significative en fonction des densités.

Concernant la croissance moyenne individuelle, le test de Newman-Keuls montre qu'il n'existe aucune différence significative ( $>0,05)$ de croissance pour une même période d'élevage selon la densité de mise en charge chez les deux espèces (Tableau 7).

Tableau 1 : Répartition des densités de mise en charge des alevins des deux espèces.

\begin{tabular}{|c|c|c|c|c|c|c|}
\hline Souche & $\begin{array}{l}\text { Familles } \\
\text { (couple) }\end{array}$ & $\begin{array}{c}\text { Densité } \\
\text { (Alevins /I) }\end{array}$ & $\begin{array}{c}\text { Volume } \\
\text { d'aquarium } \\
\text { (litres) }\end{array}$ & $\begin{array}{l}\text { Nombre } \\
\text { d'alevins }\end{array}$ & $\begin{array}{l}\text { Nombre de } \\
\text { réplicas }\end{array}$ & $\begin{array}{c}\text { Numéro } \\
\text { d'aquarium }\end{array}$ \\
\hline \multirow{12}{*}{$\begin{array}{c}C . \\
\text { gariepinus } \\
\text { (Souche } \\
\text { domestiquée } \\
\text { provenant } \\
\text { du Bénin) }\end{array}$} & \multirow{4}{*}{$\mathrm{F} 1$} & \multirow{2}{*}{4} & 50 & 200 & \multirow{2}{*}{2} & A1 \\
\hline & & & 50 & 200 & & A1 \\
\hline & & \multirow{2}{*}{2} & 50 & 100 & \multirow{2}{*}{2} & A3 \\
\hline & & & 50 & 100 & & A4 \\
\hline & \multirow{4}{*}{$\mathrm{F} 2$} & \multirow{2}{*}{4} & 50 & 200 & \multirow{2}{*}{2} & A1 \\
\hline & & & 50 & 200 & & A1 \\
\hline & & \multirow{2}{*}{2} & 50 & 100 & \multirow{2}{*}{2} & A3 \\
\hline & & & 50 & 100 & & A4 \\
\hline & \multirow{4}{*}{$\mathrm{F} 3$} & \multirow{2}{*}{4} & 50 & 200 & \multirow{2}{*}{2} & A1 \\
\hline & & & 50 & 200 & & A1 \\
\hline & & \multirow{2}{*}{2} & 50 & 100 & \multirow{2}{*}{2} & A3 \\
\hline & & & 50 & 100 & & A4 \\
\hline \multirow{3}{*}{$\begin{array}{c}C . \\
\text { anguillaris }\end{array}$} & \multirow{3}{*}{$\mathrm{F} 1$} & \multirow{3}{*}{4} & 50 & 200 & \multirow{2}{*}{2} & A1 \\
\hline & & & 50 & 200 & & A1 \\
\hline & & & 2142 & & & \\
\hline
\end{tabular}




\begin{tabular}{|c|c|c|c|c|c|c|}
\hline \multirow{10}{*}{$\begin{array}{c}\text { (Souche } \\
\text { sauvage du } \\
\text { Burkina } \\
\text { Faso) }\end{array}$} & & \multirow{2}{*}{2} & 50 & 100 & \multirow{2}{*}{2} & A3 \\
\hline & & & 50 & 100 & & A4 \\
\hline & \multirow{4}{*}{$\mathrm{F} 2$} & \multirow[b]{2}{*}{4} & 50 & 200 & \multirow{2}{*}{2} & A1 \\
\hline & & & 50 & 200 & & A1 \\
\hline & & \multirow[t]{2}{*}{2} & 50 & 100 & \multirow{2}{*}{2} & A3 \\
\hline & & & 50 & 100 & & $\mathrm{~A} 4$ \\
\hline & \multirow{4}{*}{ F3 } & & 50 & 200 & \multirow{2}{*}{2} & A 1 \\
\hline & & 4 & 50 & 200 & & A1 \\
\hline & & \multirow{2}{*}{2} & 50 & 100 & \multirow{2}{*}{2} & A3 \\
\hline & & & 50 & 100 & & A4 \\
\hline
\end{tabular}

F1 = Male $1 \times$ Femelle $1 ; F 2=$ Male $2 \times$ Femelle $2 ; F 3=$ Male $3 \times$ Femelle 3.

Tableau 2 : Composition des aliments utilisés lors de l'élevage.

\begin{tabular}{lcc}
\hline Composition de l'aliment & $\mathbf{1 ~} \mathbf{~ m m}$ & $\mathbf{1 . 8} \mathbf{~ m m}$ \\
\hline Protéiques & $57 \%$ & $55 \%$ \\
Lipides & $15 \%$ & $15 \%$ \\
Celluloses & $0,5 \%$ & $0,5 \%$ \\
Cendres & $10,5 \%$ & $11 \%$ \\
Phosphores & $1,5 \%$ & $1,6 \%$ \\
Cuivres & $8 \mathrm{mg} / \mathrm{Kg}$ & $8 \mathrm{mg} / \mathrm{Kg}$ \\
Additifs & & \multicolumn{2}{|c}{} \\
Vitamine A (U.I) & \multicolumn{2}{c}{7500} \\
Vitamine D3 (U.L) & \multicolumn{2}{c}{1125} \\
Vitamine E (mg) & \multicolumn{2}{c}{225} \\
\hline
\end{tabular}

Tableau 3 : Indices zootechniques calculés et leur mode de calcul.

\begin{tabular}{|c|c|c|}
\hline Paramètres & Formules & Références \\
\hline Gain Moyen Quotidien (GWQ) & $(\mathrm{Mf}-\mathrm{Mi}) / \mathrm{t}$ & Rukera Tabaro et al., 2005 \\
\hline $\begin{array}{l}\text { Taux de croissance spécifique pondérale } \\
\text { (TCSP) }\end{array}$ & [(LnMf-LnMi)/t] x 100 & Coulibaly et al., 2007a \\
\hline $\begin{array}{l}\text { Taux de Croissance Spécifique Linéaire } \\
\text { (TCSL) }\end{array}$ & {$[(\operatorname{lnLf}-\mathrm{LnLi}) / \mathrm{t}] \times 100$} & Coulibaly et al., 2007a \\
\hline Taux de survie $(\%)$ & $(\mathrm{Nf} / \mathrm{Ni}) \times 100$ & Rukera Tabaro et al., 2005 \\
\hline Taux de conversion alimentaire (TCA) & $\mathrm{Q} / \mathrm{G}$ & Rukera Tabaro et al., 2005 \\
\hline Coefficient de condition K (\%) & $\left(\mathrm{Pf} / \mathrm{L}^{3}\right) * 100$ & Rukera Tabaro et al., 2005 \\
\hline
\end{tabular}


Tableau 4: Variation des facteurs physico-chimique au cours de l'élevage.

\begin{tabular}{ccccc}
\hline & Température $\left({ }^{\circ} \mathbf{C}\right)$ & Oxygène $(\mathbf{p p m})$ & $\mathbf{p H}$ & Conductivité $(\boldsymbol{\mu} \mathbf{S})$ \\
\hline Matin & $29,3 \pm 0,37^{\mathrm{a}}$ & $22,13 \pm 8,98^{\mathrm{a}}$ & $7,06 \pm 0,53^{\mathrm{a}}$ & $39,63 \pm 7,34^{\mathrm{a}}$ \\
Soir & $30,08 \pm 0,52^{\mathrm{b}}$ & $32,20 \pm 13,98^{\mathrm{b}}$ & $6,81 \pm 0,36^{\mathrm{b}}$ & $45,20 \pm 3,70^{\mathrm{b}}$ \\
\hline
\end{tabular}

Tableau 5 : Comparaison des paramètres zootechniques au sein des familles pour les deux espèces. Sur une même ligne, les valeurs portant des lettres différentes sont significativement différentes (Test de Newman-Keuls, $\mathrm{p}<0,05$ ).

\begin{tabular}{ccccccc}
\hline Espèces & \multicolumn{3}{c}{ C. anguillaris } & \multicolumn{3}{c}{ C. gariepinus } \\
\hline Familles & F1 & F2 & F3 & F1 & F2 & F3 \\
\hline GMQ & $0,55 \pm 0,04^{\mathrm{b}}$ & $0,47 \pm 0,14^{\mathrm{b}}$ & $0,93 \pm 0,14^{\mathrm{ac}}$ & $1,29 \pm 0,10^{\mathrm{a}}$ & $1,38 \pm 0,22^{\mathrm{a}}$ & $1,13 \pm 0,25^{\mathrm{ac}}$ \\
TCA & $1,28 \pm 0,05^{\mathrm{a}}$ & $1,27 \pm 0,23^{\mathrm{a}}$ & $0,71 \pm 0,06^{\mathrm{b}}$ & $0,70 \pm 0,06^{\mathrm{b}}$ & $0,81 \pm 0,15^{\mathrm{b}}$ & $0,82 \pm 0,07^{\mathrm{b}}$ \\
TCSP & $9,85 \pm 0,44^{\mathrm{a}}$ & $8,08 \pm 0,91^{\mathrm{d}}$ & $10,87 \pm 0,58^{\mathrm{ab}}$ & $12,02 \pm 0,29^{\mathrm{c}}$ & $11,41 \pm 0,48 \mathrm{~b}^{\mathrm{c}}$ & $10,79 \pm 0,65^{\mathrm{ab}}$ \\
TCSL & $2,75 \pm 0,17^{\mathrm{a}}$ & $3,01 \pm 0,31^{\mathrm{a}}$ & $3,52 \pm 0,21^{\mathrm{b}}$ & $3,92 \pm 0,18^{\mathrm{b}}$ & $3,91 \pm 0,17^{\mathrm{b}}$ & $3,71 \pm 0,17^{\mathrm{b}}$ \\
K $(\%)$ & $0,84 \pm 0,07^{\mathrm{a}}$ & $0,44 \pm 0,11^{\mathrm{b}}$ & $0,92 \pm 0,02^{\mathrm{a}}$ & $0,97 \pm 0,05^{\mathrm{a}}$ & $0,85 \pm 0,02^{\mathrm{a}}$ & $0,92 \pm 0,06^{\mathrm{a}}$ \\
\hline \multicolumn{2}{c}{ GMQ $=$ Gain Moyen Quotidien, TCA = Taux de conversion alimentaire; TCSP $=$ Taux de Croissance Spécifique Pondérale }
\end{tabular}

Tableau 6 : Comparaison du taux de survie total dans chaque aquarium au cours de l'élevage.

\begin{tabular}{ccccc}
\hline & $\begin{array}{c}\mathbf{A 1} \\
\text { 4 poissons/l }\end{array}$ & $\begin{array}{c}\mathbf{A 2} \\
\mathbf{4} \text { poissons/l }\end{array}$ & $\begin{array}{c}\mathbf{A 3} \\
\mathbf{2} \text { poissons/l }\end{array}$ & $\begin{array}{c}\mathbf{A 4} \\
\mathbf{2} \text { poissons/l }\end{array}$ \\
\hline T0 & 100 & 100 & 100 & 100 \\
T10 & $82,17 \pm 7,43^{\mathrm{a}}$ & $79,83 \pm 7,55^{\mathrm{a}}$ & $87,38 \pm 14,44^{\mathrm{a}}$ & $87,69 \pm 9,39^{\mathrm{a}}$ \\
T20 & $63,92 \pm 13,32^{\mathrm{a}}$ & $66,33 \pm 9,58^{\mathrm{a}}$ & $64,17 \pm 16,99^{\mathrm{a}}$ & $76,87 \pm 10,29^{\mathrm{a}}$ \\
T30 & $53,00 \pm 13,46^{\mathrm{a}}$ & $55,75 \pm 13,66^{\mathrm{a}}$ & $60,68 \pm 16,06^{\mathrm{a}}$ & $70,10 \pm 10,72^{\mathrm{a}}$ \\
\hline
\end{tabular}

Tableau 7: Comparaison du poids moyen individuel total dans chaque aquarium au cours de l'élevage.

\begin{tabular}{ccccc}
\hline & $\mathbf{A 1}$ & $\mathbf{A 2}$ & $\mathbf{A 3}$ & $\mathbf{A 4}$ \\
& 4 poissons/l $^{\mathrm{T}}$ & 4 poissons/l $^{\text {2 poissons/l }}$ & 2 poissons/l \\
\hline T0 & $1,14 \pm 0,14^{\mathrm{a}}$ & $1,20 \pm 0,22^{\mathrm{a}}$ & $1,17 \pm 0,27^{\mathrm{a}}$ & $1,28 \pm 0,27^{\mathrm{a}}$ \\
T10 & $4,23 \pm 1,09^{\mathrm{a}}$ & $4,59 \pm 1,57^{\mathrm{a}}$ & $4,30 \pm 1,10^{\mathrm{a}}$ & $4,58 \pm 1,40^{\mathrm{a}}$ \\
T20 & $13,23 \pm 5,93^{\mathrm{a}}$ & $14,31 \pm 6,30^{\mathrm{a}}$ & $14,90 \pm 6,40^{\mathrm{a}}$ & $14,09 \pm 7,62^{\mathrm{a}}$ \\
T30 & $28,27 \pm 14,37^{\mathrm{a}}$ & $27,79 \pm 11,50^{\mathrm{a}}$ & $31,32 \pm 14,75^{\mathrm{a}}$ & $28,70 \pm 13,74^{\mathrm{a}}$ \\
\hline
\end{tabular}




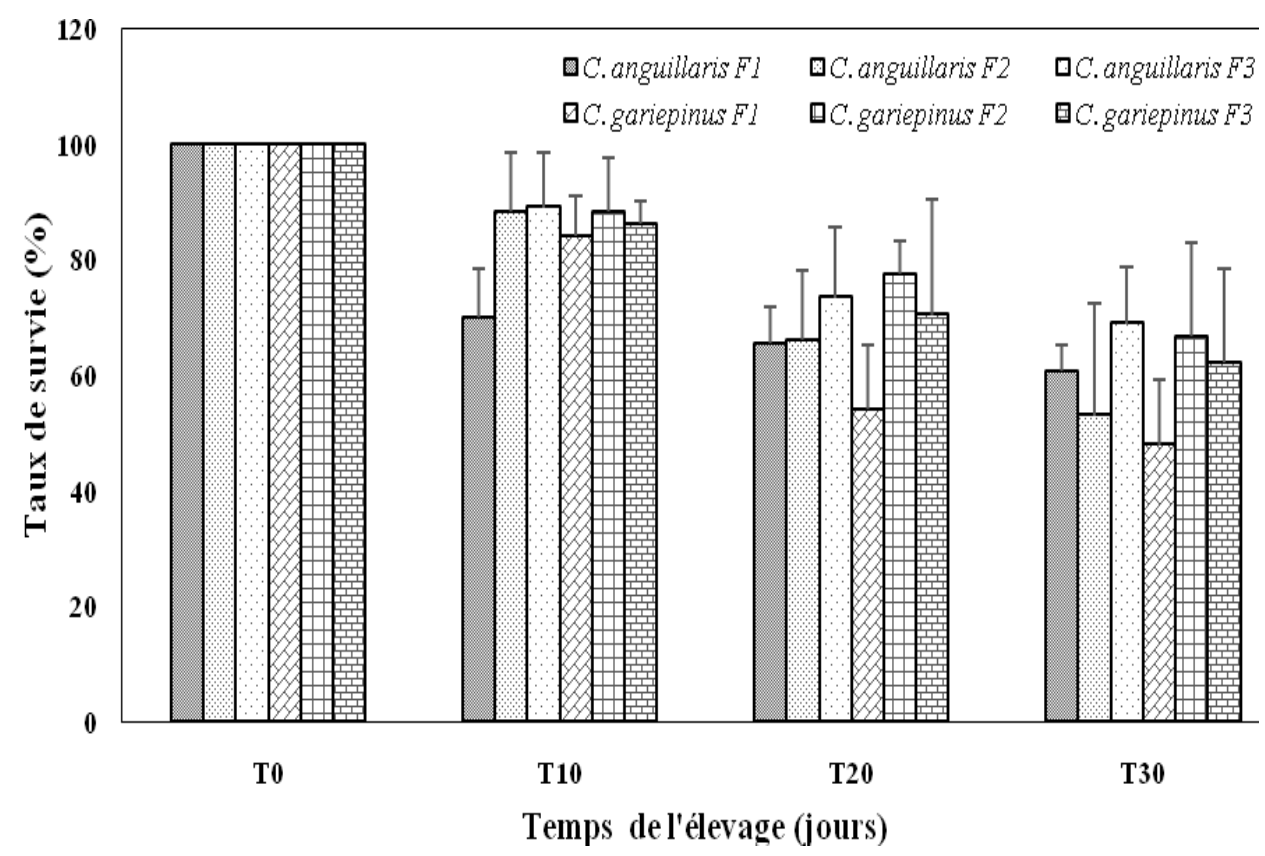

Figure 1: Courbe de l'évolution du taux de survie en fonction des familles respectivement de Clarias anguillaris (barres en pointillés) et de C. gariepinus (barres en carreaux).

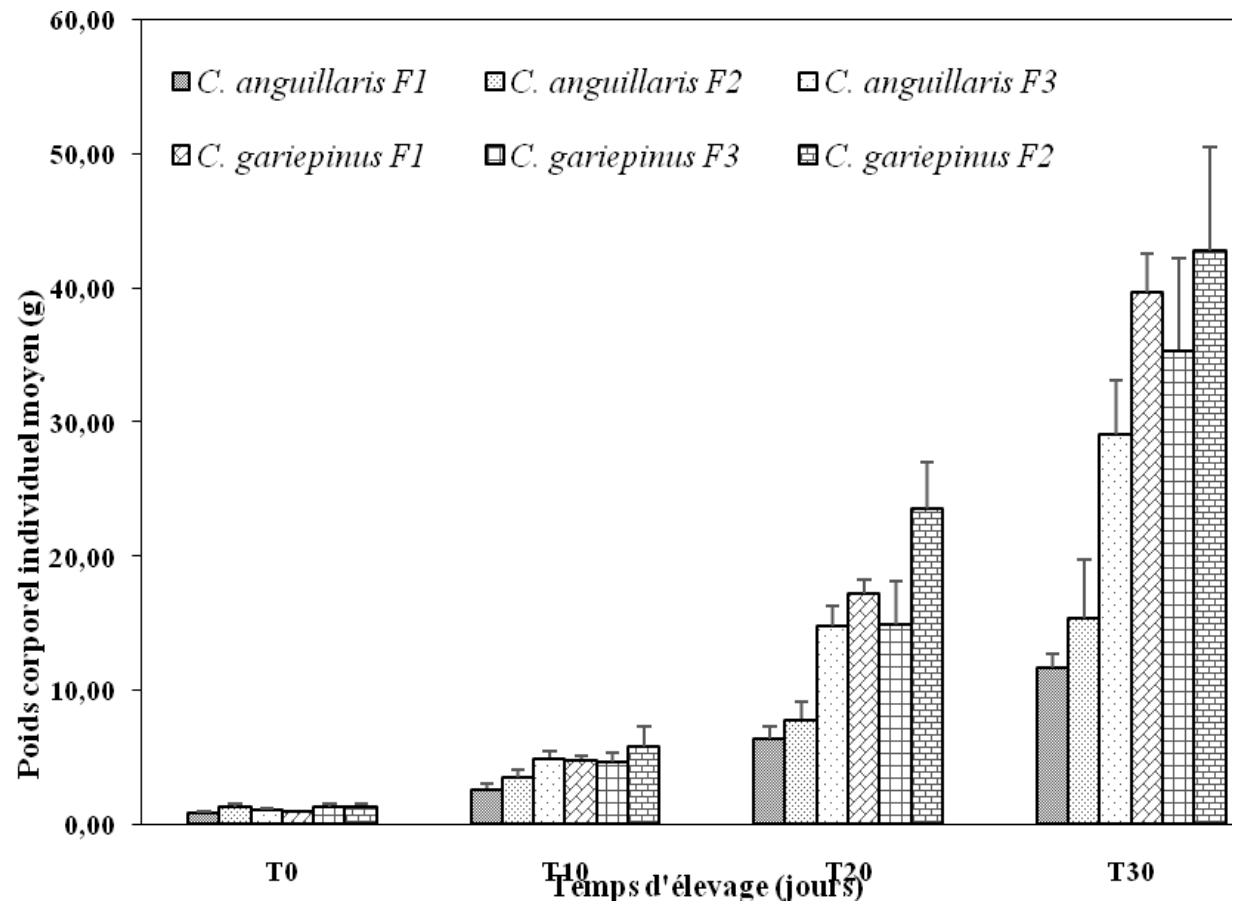

Figure 2: Courbe de l'évolution du poids moyen individuel (pmi) en fonction des familles respectivement de Clarias anguillaris (barres en pointillés) et de C. gariepinus (barres en carreaux). 


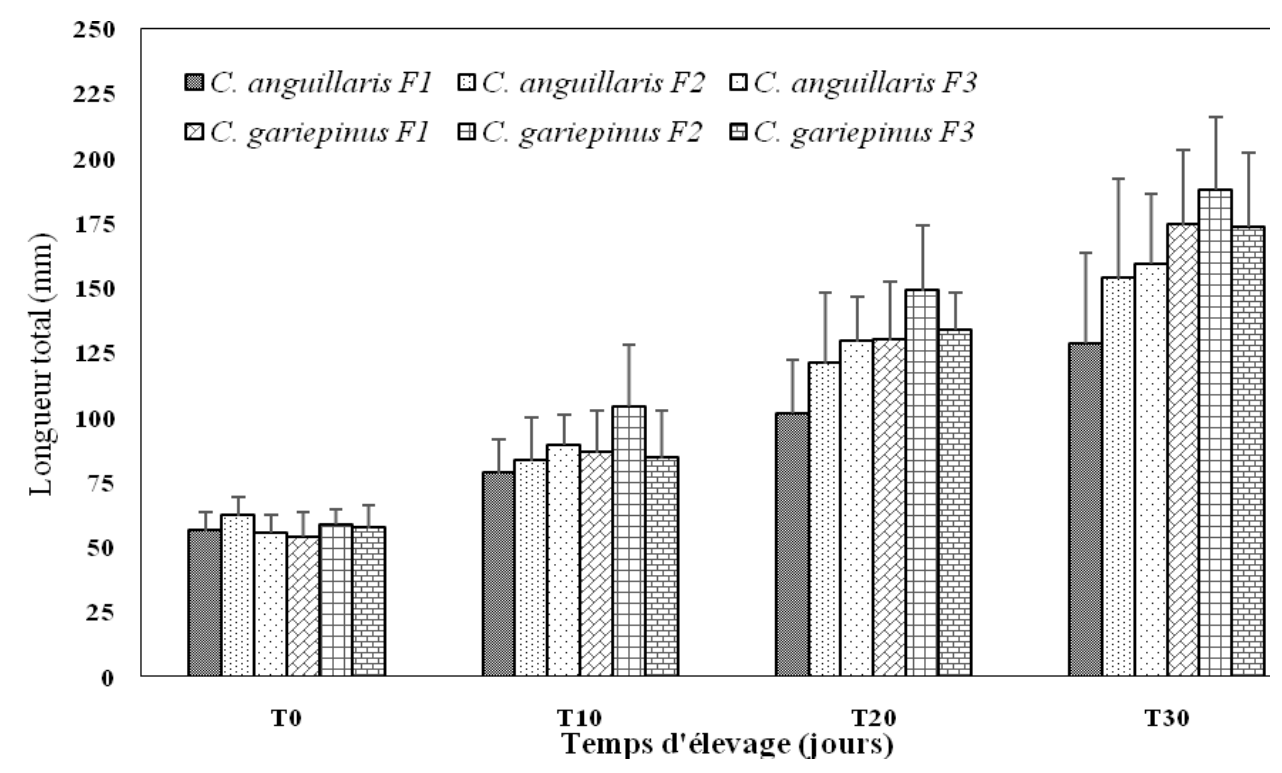

Figure 3 : Evolution de la longueur totale (LT) en fonction des familles de chaque espèce respectivement de Clarias anguillaris (barres en pointillés) et de C. gariepinus (barres en carreaux).

\section{DISCUSSION}

Les résultats de cette expérimentation n'ont révélé des différences de taux de survie qu'à l'intérieur des familles d'une même espèce mais pas entre les deux espèces. Ce qui va dans le sens des précédents travaux mais ces résultats sont meilleurs à ceux trouver par Tiogué et al. (2008) qui est de 46,26\%. Tabaro et al. (2005) ont obtenu des taux variant entre 24 et 39\% respectivement en étang simulé et en bassin, ce qui est inférieur aux résultats de cette étude. Par contre, ils sont en deçà des résultats obtenus par Akinwole et Faturori, (2007) qui est de l'ordre de 75-93\%. Il faut noter que c'est à la fin de l'expérience que les plus fortes diminutions des effectifs ont été observées et cela est en lien direct avec le cannibalisme puisque les poissons retrouvés mort présentaient des traces d'agressions. Preuve qu'ils ont été attaqués par leurs congénères. Pour certains auteurs ce cannibalisme serait une forme spéciale d'autorégulation au sein des populations qui le pratiquent (Wakano et al., 2002 ; Begon et al., 2006). Ce comportement cannibale chez les
Clariidae est surtout lié à une différence morphologique au sein d'une même cohorte (Appelbaum et Kamber, 2000). Cette différence morphologique se traduit par une variation du taux de croissance et de comportement entre les individus, certains croissant plus vite et développant une morphologie adaptée au cannibalisme, d'autres se développant normalement (Wakano, 2004 ; Solomon et Udoji, 2011). Selon plusieurs auteurs (Fontaine, 2004 ; Coulibaly et al., 2007 ; Solomon et Udoji, 2011) le cannibalisme est souvent la principale cause de mortalité en larviculture chez ces deux espèces et de façon générale chez les Clariidae et affecterait de manière très significative les taux de survie observés en élevage. Outre la différence morphologique, une compétition entre les individus induite par une forte densité favoriserait également le cannibalisme c'est-à-dire que les individus s'entre-dévorent une fois que la fréquence de rencontre est élevée (Chapman, 2000). Au cours de cette expérimentation, cela ne semble pas avoir été le cas puisqu'aucune relation n'a été trouvée 
entre le taux de survie et les densités utilisées. Toutefois, il a été montré qu'une forte densité couplée à une insuffisance des ressources alimentaires serait un facteur favorisant le cannibalisme. C'est ce qui a été constaté lors de cette étude car le cannibalisme est surtout observé la nuit lorsque les poissons se retrouvent sans apport de nourriture extérieur étant donné que l'alimentation se faisait uniquement pendant la phase diurne. Ces espèces sont reconnues pour leur forte mobilité nocturne à la recherche de la nourriture, ce qui occasionnerait de forte prédation. En résumé, ce comportement cannibale des animaux présente aussi bien des avantages que des inconvénients. En termes d'avantages, Alabi et al. (2009) citent les gains nutritionnel et énergétique, les gains de territoire pour vivre et se développer alors qu'au niveau des inconvénients, ils signalent la réduction des effectifs, la variabilité génétique de la population, ainsi la possibilité de transmission de parasites, virus et de maladies.

Cette absence de différence au niveau des taux de survie est une donnée majeure chez $C$. anguillaris, car malgré qu'elle ne soit pas encore entièrement domestiquée, les descendances se sont bien adaptées aux conditions d'élevage en captivité. Les gains moyens quotidiens obtenus au cours de cette étude sont meilleurs chez $C$. gariepinus comparativement à $C$. anguillaris. Le taux de conversion alimentaire (TCA) qui est un critère utilisé en zootechnique pour mesurer l'efficacité de la conversion d'un aliment en une production donné d'un animal montre que C. gariepinus (souche domestiquée) valoriserait mieux les aliments que $C$. anguillaris (souche sauvage). Comparativement aux précédentes études, les résultats obtenus avec $C$. gariepinus sont meilleurs que ceux de Ayinla (2007); Akinwole et Faturoti (2007) et Hecht (2013) qui sont de respectivement de 1,$3 ; 1,1$ à 1,6 et 1,1. Mais sont plus faibles que ceux enregistrés par Rukera Tabaro et al., (2005), qui ont obtenus au bout de 38 jours des taux variant entre 0,45 et 0,69 pour des juvéniles élevées en étangs.

Dans l'ensemble, les résultats de cette expérimentation révèlent un avantage comparatif de $C$. gariepinus pour l'ensemble des paramètres à l'exception du taux de survie. Toutefois une des familles (F3) de $C$. anguillaris présente des performances comparables à deux des 3 familles (F1 et F3) de $C$. gariepinus, ce qui suggère que cette espèce aurait des capacités intrinsèques intéressant et pourrait avoir les mêmes performances à la fin du processus de domestication. En effet, de précédents travaux (Cahu, 2004 ; Benhaïm, 2012) ont montré que la domestication avait des effets importants sur l'expression des performances intrinsèques des individus. C'est en ce sens que l'éthologue Konrad Lorenz cité par Goldberg (2003) décrit la domestication comme un appauvrissement des comportements sociaux spécialisés, au profit de l'hypertrophie des besoins de base comme la reproduction et l'alimentation. Le comportement social en général paraît en effet plus riche chez les animaux sauvages que chez leurs congénères de races domestiquées (Goldberg, 2003). Il a été en effet constaté au cours de l'expérimentation que les individus de $C$. anguillaris avaient des comportements sociaux différents de ceux de C. gariepinus tel que la fuite devant toute présence humaine lors du nourrissage, un regroupement de l'ensemble des individus au fond des aquariums et une prise limitée des aliments en présence de l'expérimentateur. Ce comportement était plus marqué au début de l'expérimentation. Les meilleures performances obtenues en fin d'expérimentation pourraient être plus une conséquence de ce comportement social lié à la non domestication qu'à des différences génétiques intrinsèques. Cette meilleure performance refléterait donc 
l'appauvrissement des comportements sociaux au profit de l'hypertrophie de la croissance, la souche sauvage ayant besoin de plus de temps pour opérer des changements morphoanatomiques ou morpho-physiologiques afin de s'adapter aux nouvelles conditions d'élevages.

Les densités de mise en charge de 2 et 4 poissons/litre (correspondant respectivement à 2000 et 4000 individus $/ \mathrm{m}^{3}$ ) effectuées dans cette étude n'ont pas révélé de différence en termes de gain de croissance et de taux de survie chez les deux espèces. Ces données suggèrent que ces deux espèces pourraient supporter des densités beaucoup plus supérieures, ce qui va dans le sens de fortes densités de 8500 individus $/ \mathrm{m}^{3}$ pratiquées au Nigeria en culture intensive (Hecht, 2013). Dans d'autres travaux, de meilleurs taux de survie ont été obtenus avec des densités de l'ordre de $6000-9000 / \mathrm{m}^{3}$ (Akinwole et Faturori, 2007). Il faut toutefois signaler, que si au début, aucune différence de croissance n'a été observée, des effets commençaient à apparaitre dans les aquariums avec 4 poissons/litre. Les travaux de Coulibaly et al. (2007b) sur Heterobranchus longifilis, une espèce de la même famille montrent que la croissance de celle-ci diminue avec l'augmentation des densités de mise en charge et recommande de ce fait des densités de 6 à 10 poissons $/ \mathrm{m}^{2}$ pour obtenir des meilleurs résultats pour le grossissement. L'absence de différence nette au cours de cette expérimentation concernant ce paramètre pourrait être simplement lié à la courte durée de l'élevage.

\section{Conclusion}

La présente étude réalisée sur les performances zootechniques d'une souche domestiquée de $C$. gariepinus et d'une souche sauvage de $C$. anguillaris a été réalisée dans les mêmes conditions environnementales. Les résultats obtenus sur la comparaison des performances zootechniques des deux souches sont forts intéressants. Sur la quasi-totalité des paramètres analysés, les 3 familles de la souche domestiquée de $C$. gariepinus ont présenté de meilleurs résultats comparativement à celles de $C$. anguillaris. Cependant, une des familles testées de $C$. anguillaris a présenté des caractéristiques proches à certaines familles de $C$. gariepinus. Ces résultats préliminaires montrent que $C$. anguillaris peut être utilisée en aquaculture au même titre que $C$. gariepinus. $\mathrm{Sa}$ domestication complète améliorerait sans conteste davantage ses performances zootechniques.

\section{CONFLIT D'INTERETS}

Les auteurs ne déclarent aucun conflit d'intérêts.

\section{CONTRIBUTION DES AUTEURS}

IC : Réalisateur des différentes activités qui ont conduit à la rédaction du présent article; AT: Directeur de thèse et superviseur scientifique du travail; CM, CR et $\mathrm{PK}$ sont des partenaires scientifiques et ont tous participé à l'élaboration du protocole expérimental et à la lecture de l'article.

\section{REFERENCES}

Akinwole AO, Faturoti EO. 2007. Biological performance of African catfish (Clarias gariepinus) cultured in recirculating system in Ibadan. Aquaculture Engineering, 36: 18-23.

Alabi T, Patiny S, Verheggen F, Francis F, Haubruge E. 2009. Origine et évolution du cannibalisme dans les populations animales : pourquoi manger son semblable? Biotechnol. Agron. Soc. Environ., 13(3): 409-425.

Appelbaum S, Kamker E. 2000. Survival, growth, metabolism and behaviour of Clarias gariepinus (Burchell 1822) early stages under different light conditions. Aquacult. Eng., 22: 269-287. 
Atse BC, Koffi KM, Konan KJ, N'dri KM. 2012. Effets du taux de rationnement et de la fréquence de tri sur la croissance, la survie larvaire et le cannibalisme chez le silure Heterobranchus longifilis Valenciennes, 1840 J. Appl. Biosci., 59: 4358-4365.

Ayinla OA. 2007. Analysis of feeds and fertilizers for sustainable aquaculture development in Nigeria. In Study and Analysis of Feeds and Fertilizers for Sustainable Aquaculture Development, Hasan MR, Hecht T, De Silva SS, Tacon AGJ (eds). FAO Fisheries Technical Paper No. 497: Rome, FAO; 453-470.

Begon M, Townsend CR, Harper JC. 2006. Ecology: From Individuals to Ecosystems (4th edition). Blackwell Publishing: Oxford, UK.

Benhaïm D. 2012. Caractérisation de l'adaptation comportementale des téléostéens en élevage : plasticité et effets de la domestication. Thèse de doctorat, Université la Rochelle, Rochelle, p 241.

Cahu C. 2004. Domestication et fonction nutrition chez les poissons. INRA Prod. Anim., 17: 205-210.

Chapman JW, Trevor W, Martinez AM, Cisneros J, Caballero P, Cave RD, Goulson D. 2000. Does cannibalism in Spodoptera frugiperda reduce the risk of predation? Behav. Ecol. Sociobiology, 48: 321-327.

Coulibaly A, Koné T, Ouattara NI, N'Douba V, Snoeks J, Kouamélan EP, Bi GG. 2007a. Evaluation de l'effet d'un système de refuge sur la survie et la croissance des alevins de Heterobranchus longifilis élevés en cage flottante. Belg. J. Zool., 137(2): 157-164.

Coulibaly A, Ouattara IN, Koné T, N'douba V, Snoeks J, Bi G.G, Kouamélan EP. 2007b. First results of floating cage culture of the African catfish Heterobranchus longifilis Valenciennes,
1840: Effect of stocking density on survival and growth rates. Aquaculture, 263: 61-67.

De Graaf GJ, Janssen JAL. 1996. Artificial reproduction and pond rearing of the African catfish, Clarias gariepinus in sub-Saharan Africa -A handbook. FAO, Fisheries Technical Paper, No 362. Rome, FAO, 73 p.

Ducarme C, Micha JC. 2003. Technique de production intensive du poisson chat africain, Clarias gariepinus. Tropicultura, 2: 189-198.

Eding EH, Kamstra A. 2002. Netherlands farms tune recirculation systems to production of varied species. Global Aquaculture Advocate, 5: 52-54.

Eding EH, Kamstra A, Verreth JAJ, Huisman EA, Klapwijk A. 2006. Design and operation of nitrifying trickling filters in recirculating aquaculture: A review. Aquaculture Engineering., 34: 234-260.

FAO. 2012. La situation mondiale des pêches et de l'aquaculture. Département des pêches et de l'aquaculture de la FAO. Organisation des Nations Unies pour l'Alimentation et l'Agriculture, Rome. p 261.

Fontaine P. 2004. Premiers résultats en Perciculture hors-sol chez Lucas Perche. Aquafilia., 2: 10-14.

Fontaine P, Le bail PY. 2004. Domestication et croissance chez les poissons. INRA Prod. Anim., 17(3): 217-225.

Goldberg J. 2003. « Domestication et comportement ». Bulletin de la Société Zoologique de France, 128(4): 89.

Hecht T. 2013. A review of on-farm feed management practices for North African catfish (Clarias gariepinus) in subSaharan Africa. In On-Farm Feeding and Feed Management in Aquaculture, Hasan MR, New MB (eds). FAO Fisheries and Aquaculture Technical Paper No. 583: Rome, FAO; 463-479. 
Hossain MAR, Haylor GS, Beveridge MCM. 2001. Effect of feeding time and frequency on the growth and feed utilization of African catfish Clarias gariepinus (Burchell 1822) fingerlings. Aquaculture Research, 32: 999-1004.

Paugy D, Lévêque C, Teugels G.G. 2003. Faune des Poissons d'Eaux Douces et Saumâtres de l'Afrique de l'Ouest (tome 1), Stiassny MLJ, Teugels GG, Hopkins CD. IRD: Paris.

Ramade F. 2001. Dictionnaire de l'écologie Encyclopaedia Universalis. Albin Michel: Paris.

Rukera-Tabaro S, Micha JC, Ducarme C. 2005. Essais d'adaptation de production massive de juvéniles de Clarias gariepinus en conditions rurales. Tropicultura, 23: 231-244.

Solomon RJ, Udoji FC. 2011. Canibalism Among Cultured African Catfishes
(Heterbranchus longifillis And Clarias gariepinus). Nature and Science, 9(9): 113.

Tiogué TC, Nguenga D, Tomedi MTE, Tchoumboue J. 2008. Quelques performances reproductives et taux de survie de deux souches du poisson-chat africain Clarias gariepinus (Burchell, 1822 ) et de leurs croisés à KoupaMatapit. Int. J. Biol. Chem. Sci., 2: 469477.

Wakano JY, Kohmatsu Y, Yamamura N. 2002. Evolutionary dynamics of frequency-dependent growth strategy in cannibalistic amphibians. Evol. Ecol. Res., 4: 719-736.

Wakano JY. 2004. Drastic growth effect may explain sympatric cannibalistic polymorphism. J. Theor. Biol., 226: 6977. 